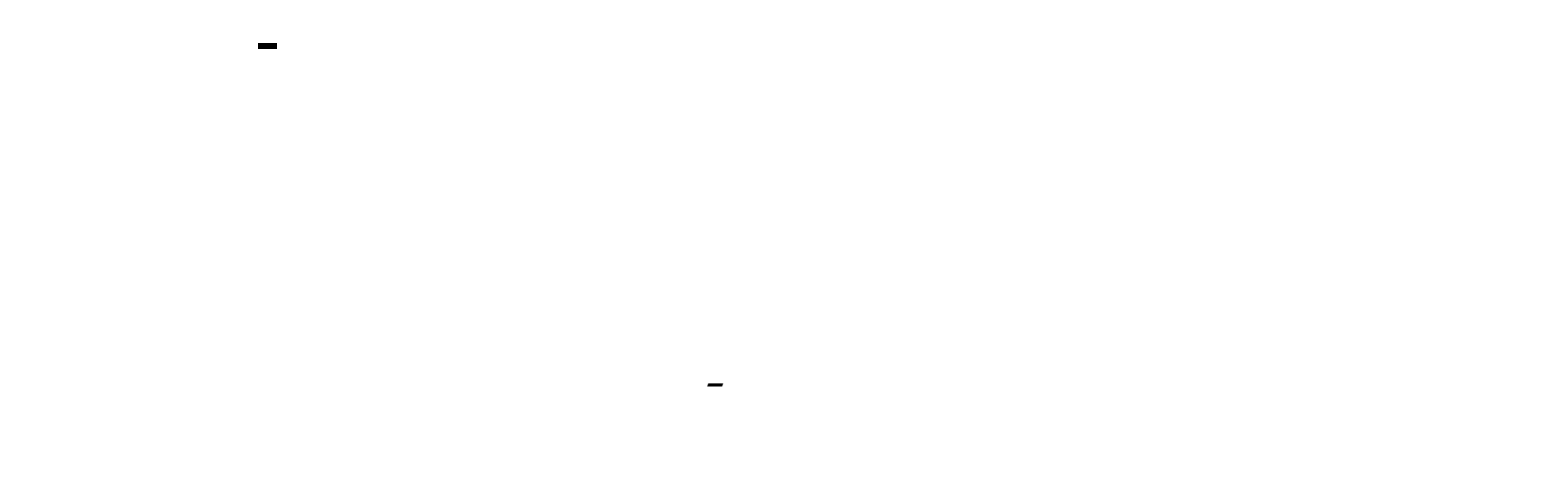

(تاريخ الاستلام 2002/12/24 ، تاريخ القبول 2002/6/12)

\title{
المالضص
}

حددت ملامح الطبقية الحياتية والمناحي الظورية للفترة الانقالية بين العصر الطبلثيري العلوي -

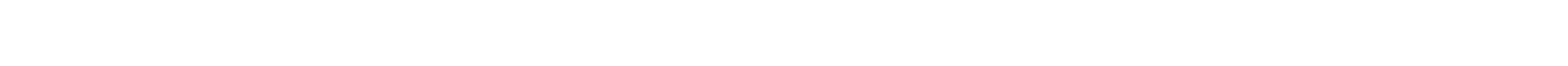
الشكلية لانواعمن الاوسترلكودا تعود للاجنلس :

Anommatocythere; Brachycythere; Buntonia; Kaesleria; Mehsella; Nucleolina and Protobuntonia .

ان الدلال المسقة من درلسة الاوسترلكودا للفترة اعلاه تشير الهى القارب الثكلي الكبير بين افراد هذه المجموعة فضلًا عن الاقراض المفلجئ لافراد الاجناس: Brachycythere; Kaesleria; Protobuntonia .

على الحد الفاطل بين العصر الطبلثيري العلوي والعصر الثلاثي اللسفل ، ولس تـمر اف ـراد الاجذ ـلس: Buntonia, Mehsella; Nucleolina - Anommatocythere

\section{Distribution and Evolutionary Trends of The Upper Cretaceous- Lower Tertiary Ostracode From Iraq and Arabian Gulf.}

\author{
Nisreen M. Aziz \\ Department of Geology \\ College of Science \\ Mosul University
}

\section{ABSTRACT}

The Ostracode biostratigraphic evidence and the evolutionary trend of Upper Cretaccous-Lower Tertiary Ostracodes are determinated by using the vertical and lateral distribution and the morphological change of the selective genera and species. 
Anommatocythere; Brachycythere; Buntonia; Kaesleria; Mehsella; Nucleolina and Protobuntonia.

The evidence determinated by the study of the above taxa of this time showes closely resemblance in their morphalogical features and suddenly disappears during the Upper Cretaceous of the genera:

Brachycythere; Kaesleria; Mehsella; Protobuntonia and other species continue in the Lower Tertiary, Buntonia; Mehsella and Nucleolina. Otherwise species of Anommatocythere show first appear during the Lower Tertiary .

\section{اللقدمة}

لظهرت العديد من لجنلس وانواع الاوسترلكودا للعصر الطبلثيري العلوي والعصر الثلاثي الاشفل

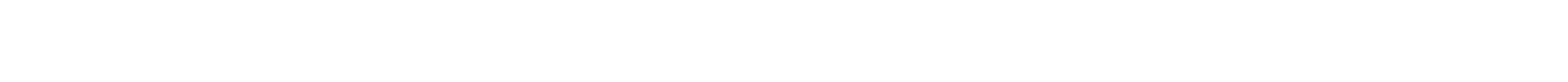
كن الانتشار الجغرافي الولسع لاغلب افرادها، ان العيدمن التغيرات البيئية الفجائية والتدريجية يمكن ان ان

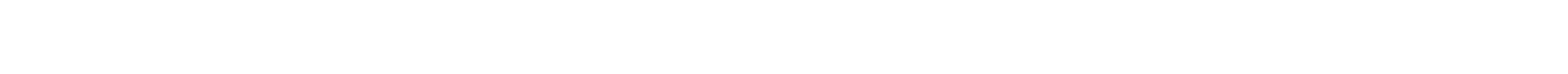

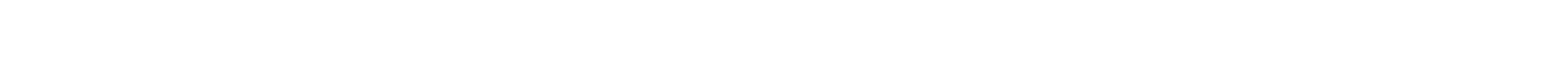

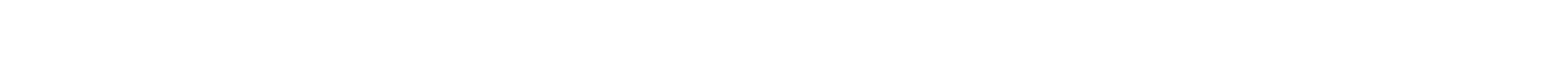

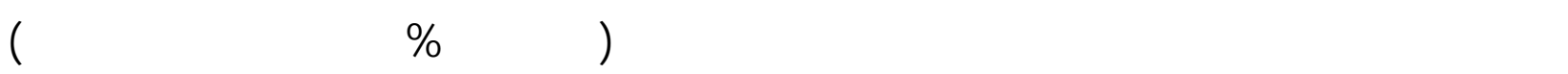

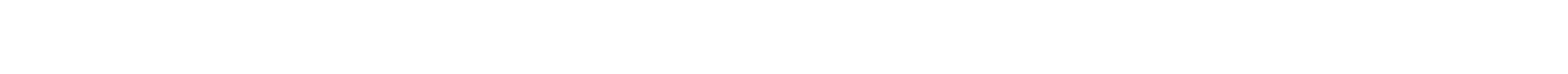
للبحر العمق اليضاً. ان هذه الاقراض يكونشائع اليضاً في اوسترلكودا الميه الضحلة ولم تحدث تغيرات ظورية قوية خلل تلك الفترة وانما تحددت في لجنلس والنواعمعينةسيم التركيز عليها خلل البحث الحالي.

\section{الانتشار اللابلي للاوسترلكودا}

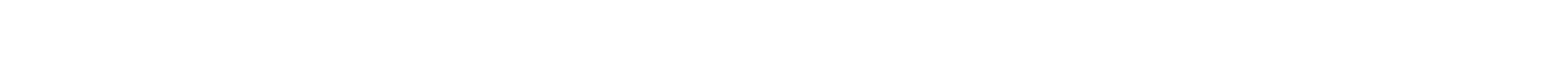

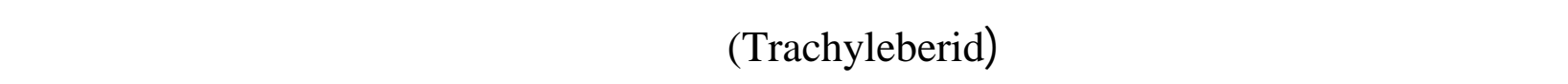

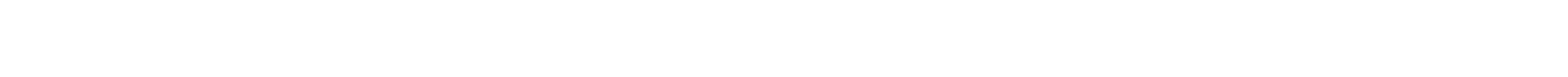
الضحلة.

ان عملية تحديد الاققراضلت في مجلمبع الاوسترلكودا يمكن ان تكون علمل مساعد في هذا للسيق

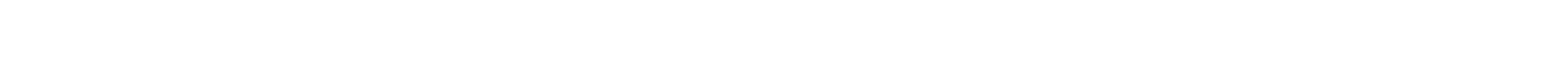

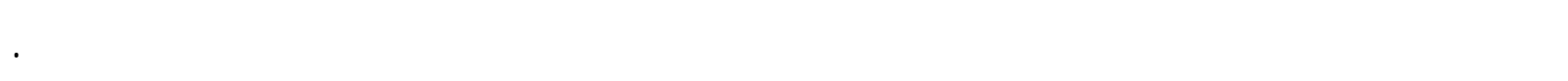


وصف هذا الجنس لاول مرة من منطلق لمريكا (Howe,1935 ) من تسسبك الايوسين انمونج ة

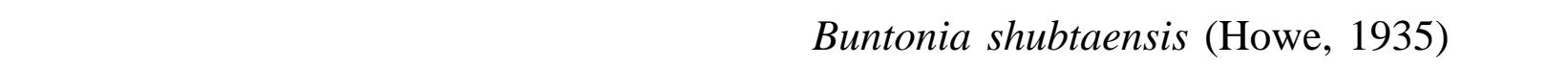
لمنطق لمريكا ،اوربا ، افريقيا ولسيا من العصر الطبلثيري ولحد الآن .

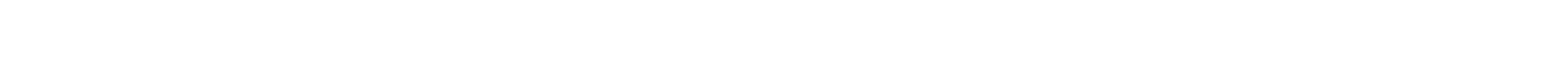
B. cretacea

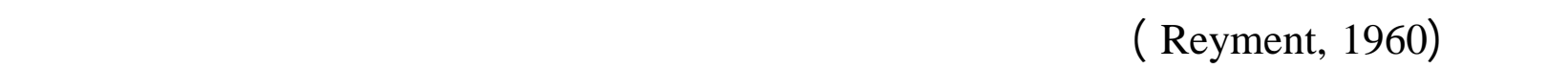
الولسع حيثسبل (Apostolescu,1963) تولجستة النواع تعود لهذا الجس المنطق المنينغل .

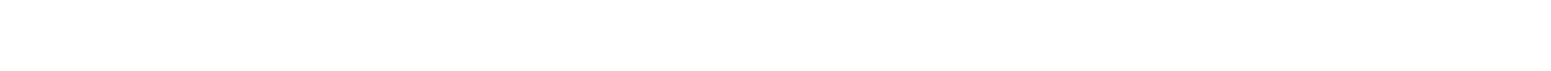

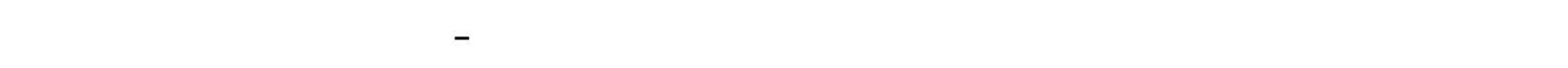
. B. cretacea (Al- Ubide, 1989)

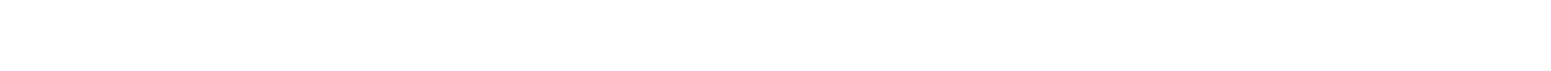
الكونيلسي (Grosdidier, 1973) ممثلاً بتولجد الانواع.

B. IRC33 Grosdidier 1973

B. IRE9 Grosdidier 1973

وسجل تولجد افراد لهذا الجنس في منلطق عملن (Athersuch, 1988 ) من الكلمبلني -الملسترختي

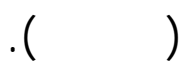
على العموم تنوع والنتشار افراد هذا الجنس يكون قليلاً لمنطق العراق والخل بـج العرب مي خ ـلالل

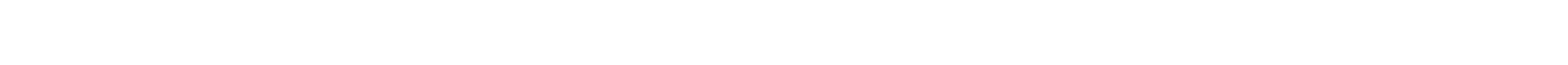
العراقسبل لمل من (Yousif, 1987, Aziz, 2001) الانواع التالية تباعَا.

Buntonia aalijensis Aziz, 2001

Buntonia senouensis Apostolescu, 1963

B. $\quad$ khurmalenis Aziz, 2001

B. desertensis Aziz, 2001

B. $\quad$ sp.1 yousif, 1987

B. jordanica Bassiouni, 1969 
每

شكل ا: المديت الطباقية لأنواع الاوسترلكودا ذات الأهمية الطباقية المميزة خلل العصر الطبلثيري والعصر الثلاثي في منططق العراق والخليج العربي

AD : Abu Dhabi, Irq : Iraq, Irn : Iran, O: Oman, SA : Saudia Arabia 


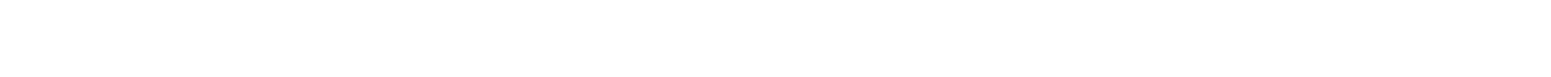

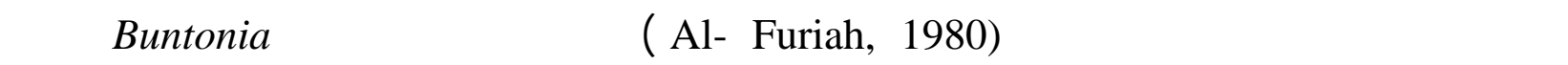
المنطاق.

\section{:Brachycythere}

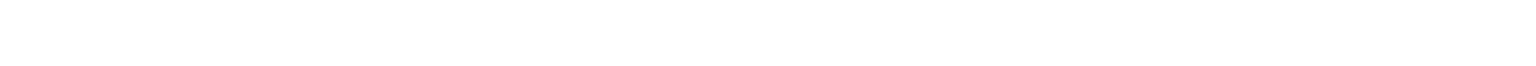
Cythere sphenoides (Reuss,1854) من Brachycythere (B.) sphensides (Reuss) انتشارَ وتنوعَ ولسعين لمختلف منططق العالم خلل العصر الطبلثيري. يعود اقدمسجل لتولجد افراد هذا الجنس اله منلطق غرب افريقيا من منطاق الكابون من المنذوماني

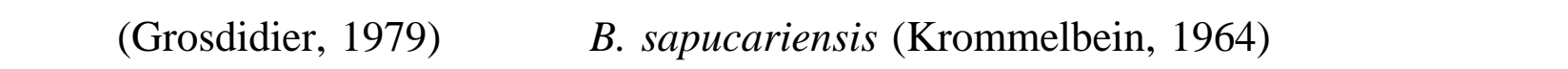
ونسل تولجد افراد الجنس في منلطق لخرى في افريقيا خلال فت _رات لاحق مة (Bismuth et al., 1981) (Grekoff, 1951, 1969)

B.hatraensis ما في منطق العراق فلقم ظهور لهذا الجنس يعود الم الكونيلسي ممثلاً بـ النوع

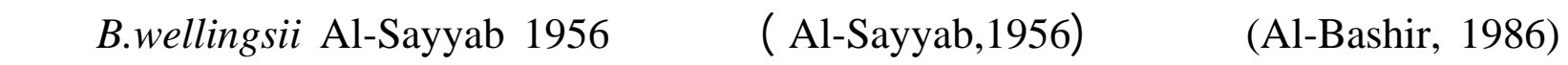

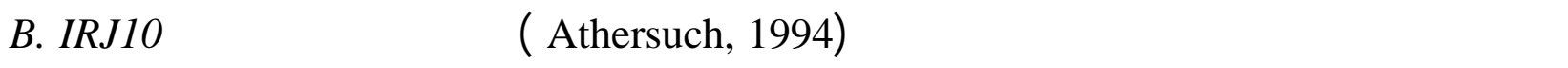
(Grosdidier, 1973)

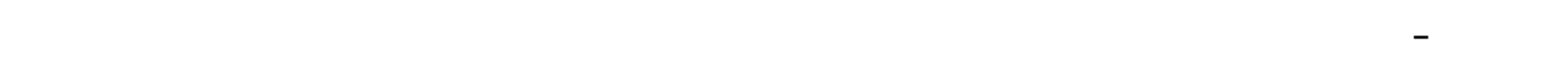
. (Grosdidier, 1973)

لما في منطق الخليج العربي الاخرى فقد لظهر تنوعاَ وانتثد ارأ ولس عيين لمذ لطق اللد عودية

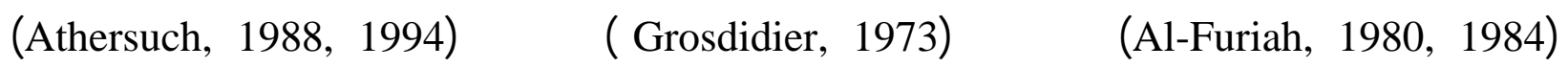
والبوظبي (Athersuch, 1987). لن هذا الاختلف في بدايةظهور افراد هذا الجنس بين منطلق غرب وشمل افريقيا ومنطق الخلبج العربي والعراق يططي دلالة واضحة على اتجه الهجرة المحتملة من منطاق غرب افريقيا ال مع من لطاق شمل افريقيا واله منطلق الخلبج العربي (Al- Ubide, 1989).

\section{Mehsella}

وصف هذا الجنس لاول مرة من قل ( Reyment, 1960 ) من الطبلثيري العلوي -العصر الثلاثي

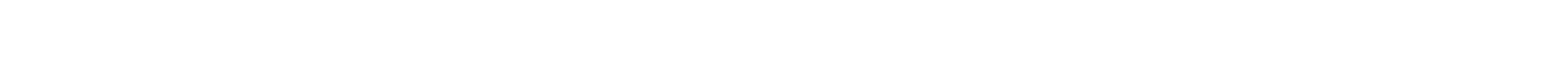

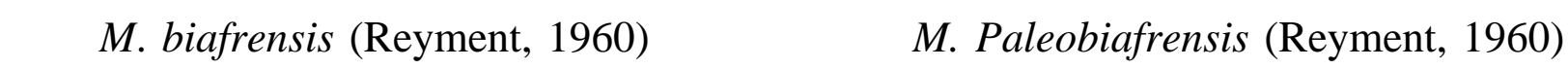
الايوسين م - ن نيجي - -ريا كم ا وصف (Ware, 1992) النوع M. africana. a الايوسين في ليبيا. 
لما في منطلق الخلبج العربي فلم يقم تسجيله الا في منطلق ايران (Grosdidier,1973 ) من الطبلثد يـري

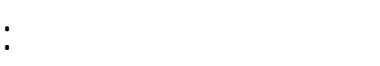
Mehsella IRE17 Grosdidier, 1973
M. IRJ $1 \quad$ Grosdidier, 1973
M. IRE31 Grosdidier, 1973
M. IRC23 Grosdidier, 1973
M. IRE30 Grosdidier, 1973

من الطبلثيري العلوي والتي ظهرت انواع منها نقاربًا كبيرًا لانواع تعود للجنس Kaeslera منها الذ ـوع M.IRC23 والذي وصفه (Athersuch, 1994 تهت تسمية Kaesteria IRC23 عملن كماسجله (Babinot and Bourdillon de-Grissac,1987) وكذك (Athersuch 1987) aن عمان

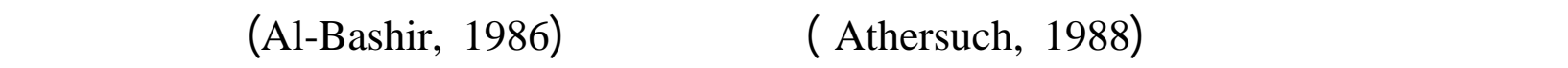
Brachycythere gulaisamaensis بينهما.

على العمو افراد هذا الجنس تشبه اله حد بعيد افراد الجنس Brachycythere في اللث ــل الع لما والانفاخ الطني ويطي هذا القارب الشكلي نوع من الاتجاهلت الظويرية.

\section{:Kaesleria}

وصف هذا الجنس لاولمرة من قبل (Al-Furiah, 1986 ) من الكلمباني -الملسترخت لمذ طلق

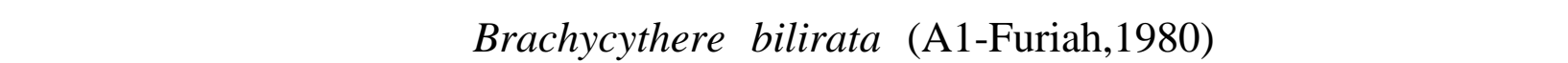
وتنوعَا ولسعين لمنطقق للسعوية ممثلاً بالعديد من الانواع لشكل () ).

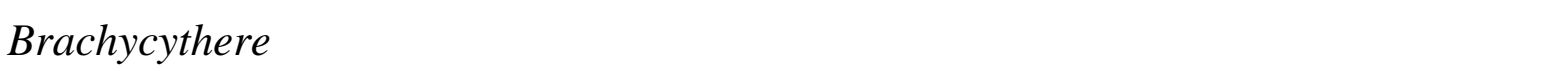
(الا اله يختلف في المتلكه لزخرفةشبكية واضحة وقعه عينية واضحة ،وفي عدد (Alexander, 1933)

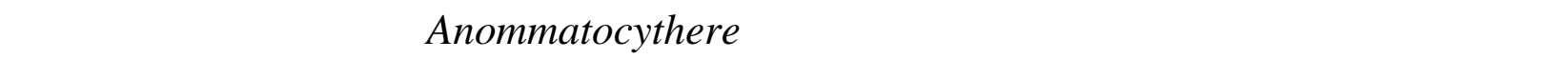
شكل الزاوية الرلسية الخلفية ،وفي كون النهاية الاملمية التل النضغطاً وفقدان الحلجز الطني والذي بدوره

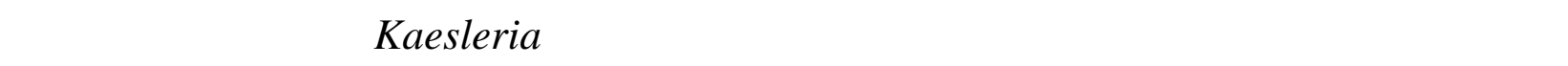

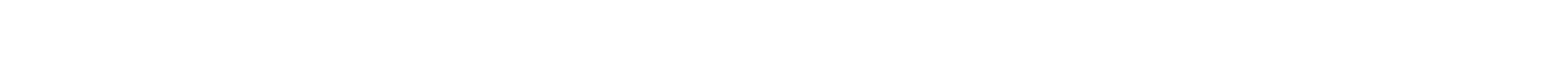
الطبلثيري العلوي اله العصر الثلاثي اللسفل ، كما وظظهرت اغلب افرادها مديلت جيولوجي ـة قص يرة لتطي اهمية طباقية مميزة .

ووصف (Grosdidier, 1973 ) النوع Mehsella IRE31 من الكلمباني الاعل ق -الملس ـترختي

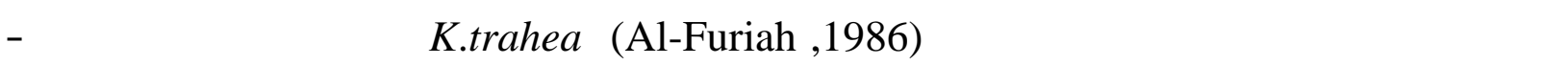

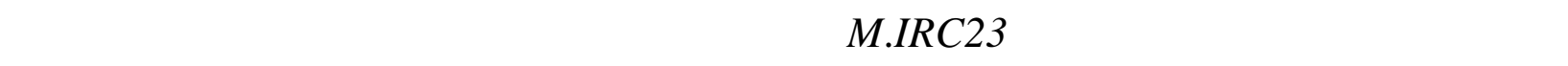
من الملسترغي لمنطق المعونية. K.undulata (Al- Furiah,1986) 
على العموم افراد الجن Kaesleria تختلف عن افراد الجن Mehsella في درجة قطور القعة

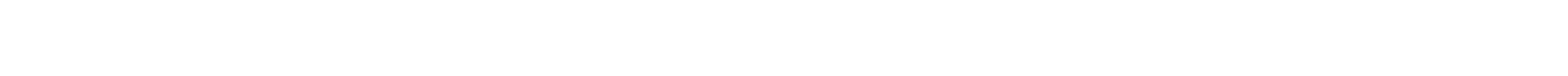
الكلمباني الاعلى لمنطق السعوية (Al-Furiah, 1986) .

\section{:Protobuntonia}

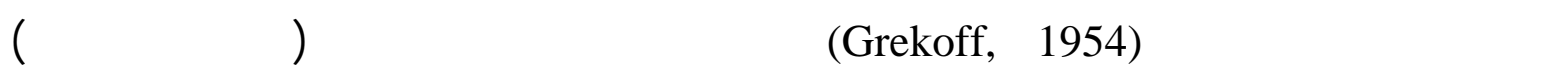

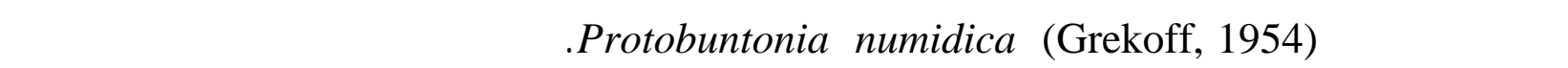

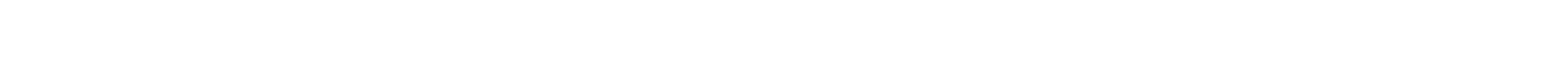

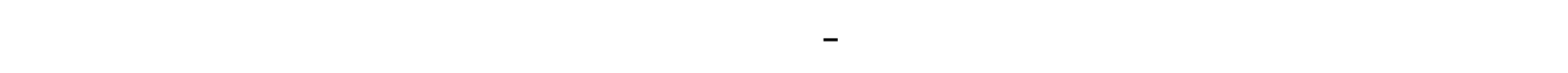

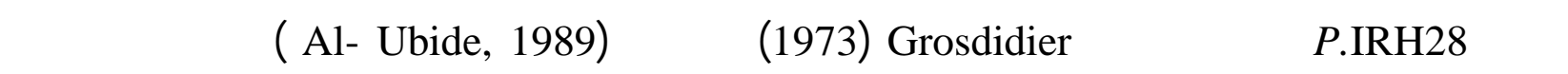

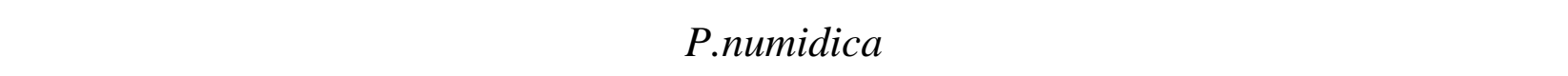

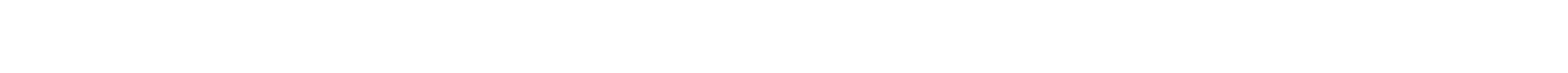

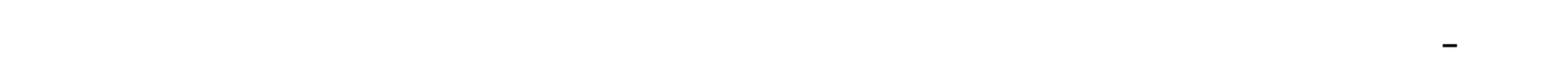

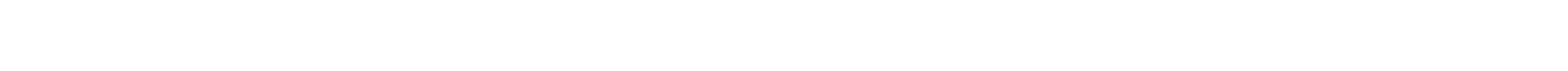
دالة لفترة الطبلثيري العلوي.افراد الجن بuntonia (Howe, 1935) Brachycythere (Alexander, 1933) في الشكل العلم وطبيعة الزخرفة الا ان الاختلاف والتغاير يكون

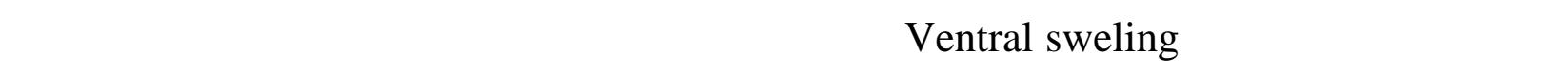

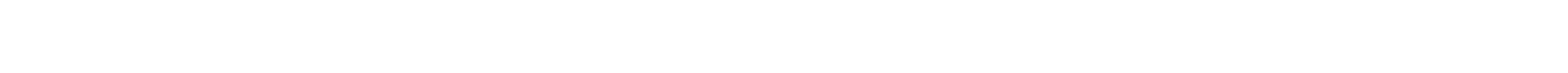
هذا الجنس خلل الطبلشيري العلوي.

\section{الجض Nucleolina:}

وصف هذا الجنس لاول مرة من الباليوسين لمنطق مالي من قل (Apostolescu, 1961) المونجة

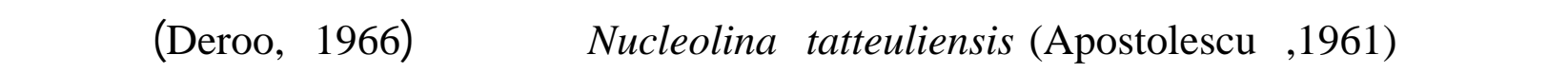
N. tenuiornata

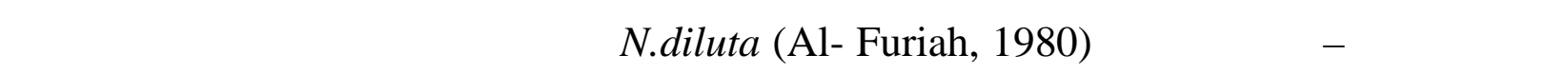

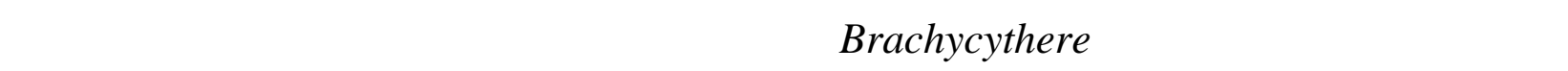

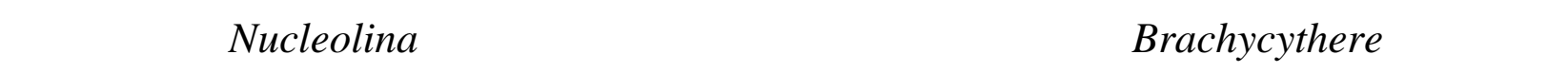

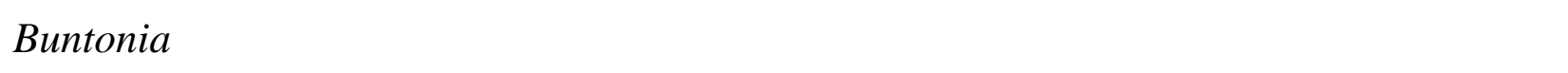

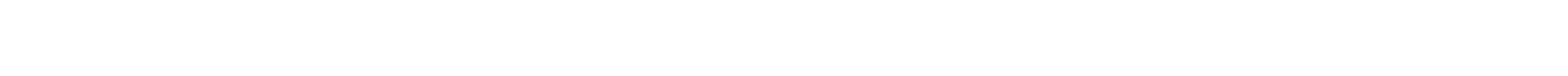

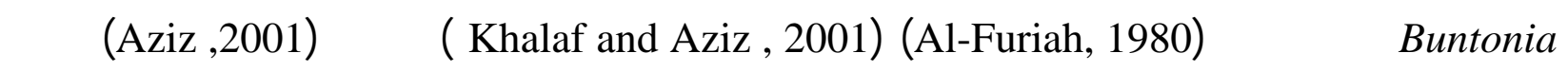


تعود لجن Buntonia تعطي نقاربًا في للثكل العلم لافراد الجس Nucleolina الا ان الاختلاف يكون في النهايتين الاملمية والخفية للدرع حيث تكون مستدقة ومضغوطة بشكل واضضح جداً.

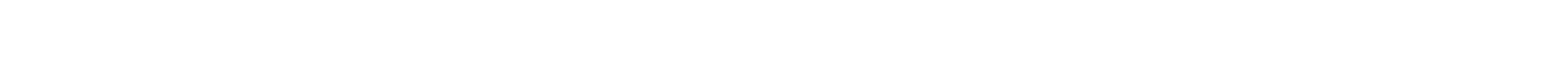

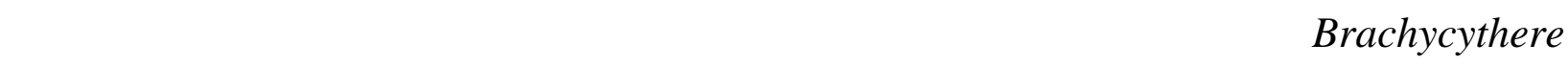
Nucleolina (الملسترختي الاعله). (افوي)

\section{Anommatocythere}

وصف لاول مرة من قل (Sohn, 1970) من الايوسن الإفل امنطاق البلكستن المونجة المثالي A. microreticulata (Sohn, 1970) (Al-Furiah and Siddiqui, 1981) وللسعودية (Aziddiqui,1971)

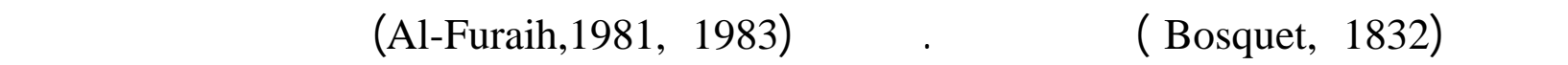
اللسعوية.

وضع كل من (Al-Furaih and Siddiqui, 1981) الجنس Anommatocythere تص نيفاً تح ـت (Tribe Echinocythere Hazel, 1967) العضلية.

يختلف افراد الجنس Anommatocythere عن الجنس Brachycythere في كون الانفاخ الطن ي أقل طوراً وفقدان العقة اللثبه وسطية والققة العينية والانحدار للشديد للحافة الظهرية.

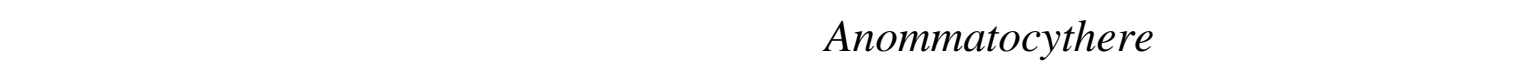

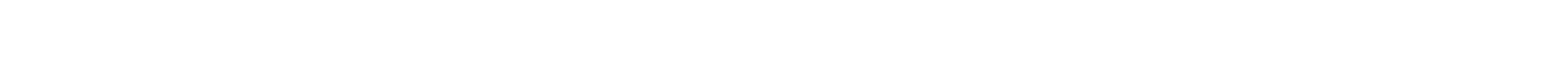
وعلاقتها القوية باوراد الجنس Brachycythere والذي اقرض وضجون بنهاية العصر الطبلثيري.

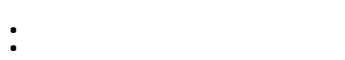

حثت العديد من الاتجاهات الظورية خلل العصر الطبلثيري الاعلى -العصر الثلاث مي الله فل

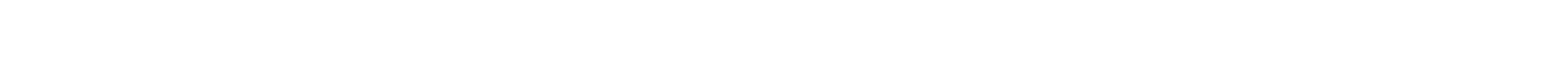

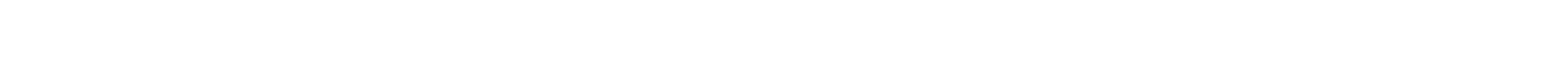

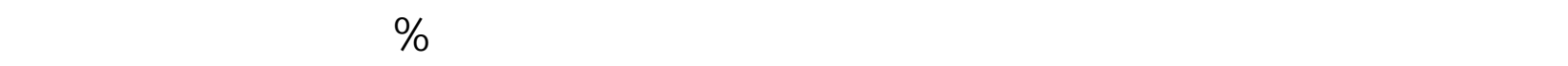

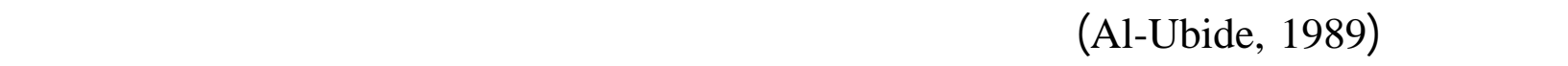

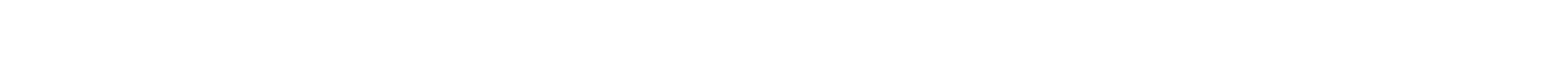
التغيرات في الصفل الثكلية التدريجية ( Gradual Divergence ) خلال الفترة الكلمباني -الملس تحرختي لمنطلق العراق والخلبج العربي (للسلحل الجنوبي لبحر التيش بصورة التبلة علمة). 


\section{من خلال درلسة التوزيع الطبالقي للاجنلس:}

Buntonia; Brachycythere; Mehsella; Kaesleria; Protobuntonia; Nucleolina and Anommatocythere.

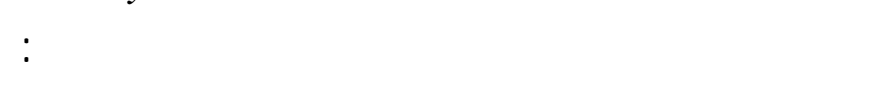

- شكل المؤخرة يصح لكثرلمتدارة خلال الملسترختي - الباليوسين .

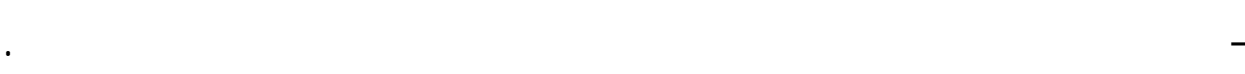

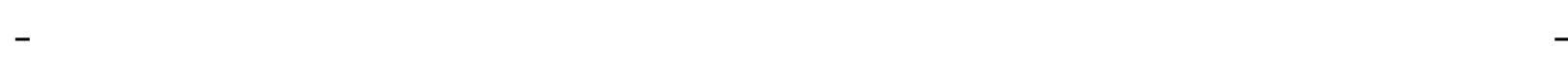

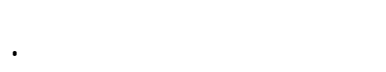

اقدمسبل لتولجد افراد الجن Brachycythere يعود اللى للسنوماني والذي التصف بصدفةطولانية

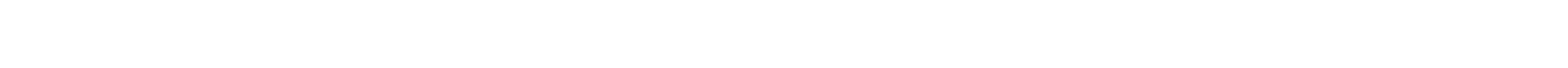

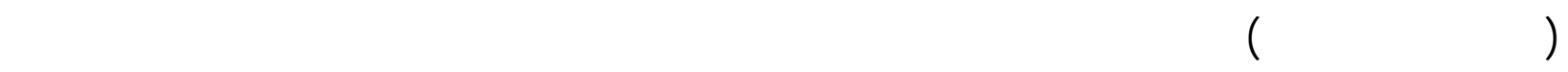

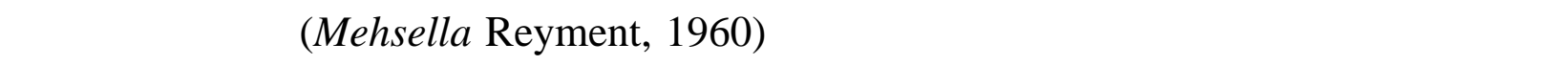

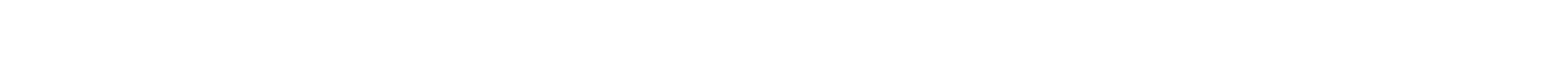

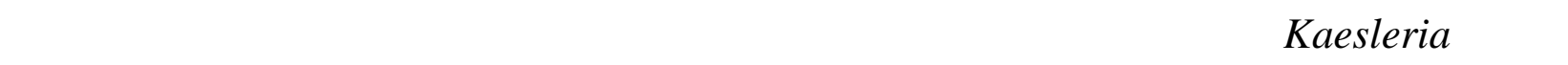

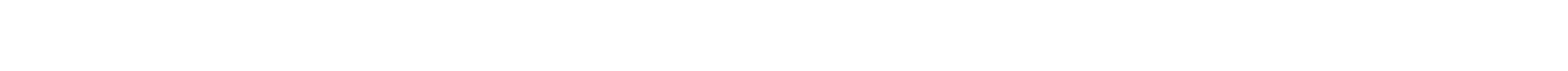
الشكلي الكبير بين افراد جنس Brachycythere والجنس Kaesleria تنطي دلالة واضحة على الت ـرلبط الظوري الوالضح بينهما.

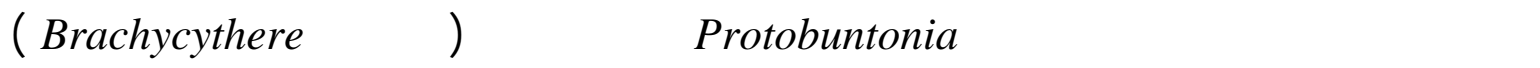

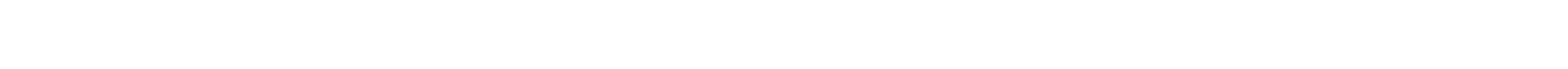
أفراد هذا الجنس تكون اقرب لجن Brachycythere من افراد الجنس Buntonia . وخلل الملسترختي ظورت لشكل تعود للجنس Brachycythere من خلل زيادة لستدارة النهاي ة

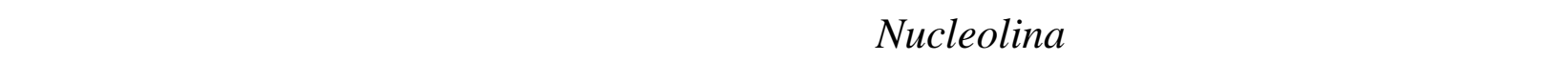

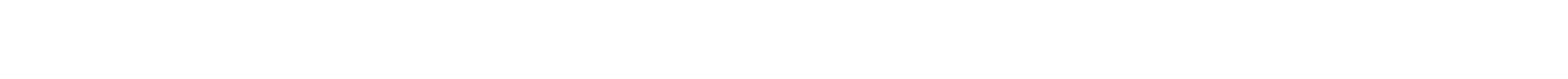

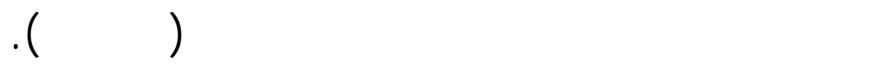

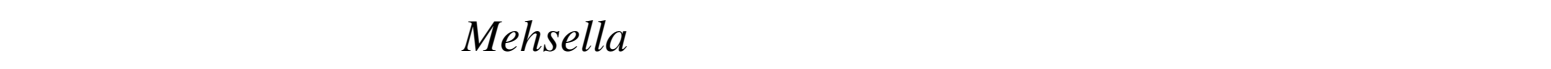

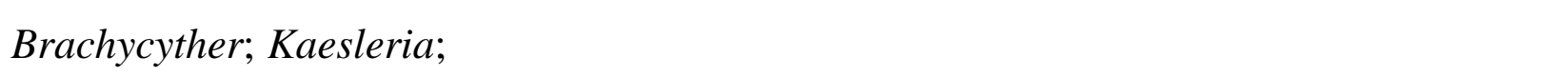

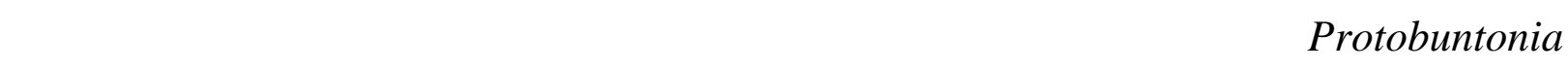

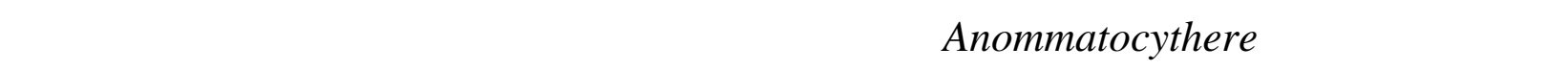

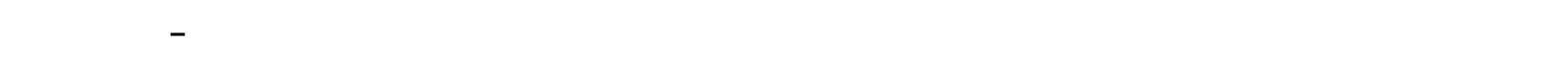
المنطق العراق والخلبج العربي. 
每

شكل 2: المنلهي الظورية لعددمن لجننلس الأوسترلكودا خلل العصر الطبلثيري العلوي -العصر

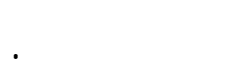

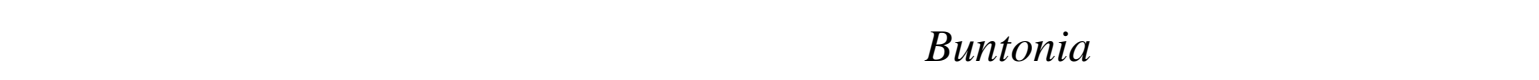

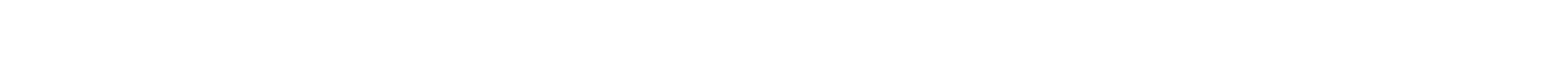




\section{الاثشكل المطورة خلل العصر الثلاثي الاسفل ظور انمط من الزخرفة اللثبكية وضيق النهاية الخلفي ة}

\section{REFERENCES}

Al-Bashir, J.M.T., 1986. Cretaceous Ostracode of the Superfamily Cytheracea from Iraq. Their biostrotigraphy and correlation with adjacent regions. Ph.D. unpub. Thesis, 586p.

Al-Bashir, J.M.T., 1995b. Glenocythere? rumilaiensis a new ostracode species from the Ahmadi Fromation (Lower Cenomanian) of Iraq. 5th Jour. Geol. Conf \& Geoecone III, pp.552-560.

Alexander, C.I., 1933. Shell structure of the Ostracode genus Cytheropteron and fossil species from the Cretaceous of Texas. J. paleontol., 7(2), pp.181-214.

Al-Furiah, A.A.F., 1980. Upper Cretaceous and Lower Tertiary Ostrocode (Superfamily Cytheracea) from Saudi Arabia. Univ. Riyath, Saudi Arabia, 211p.

Al-Furiah, A.A.F., 1983b. Middle Cretaceous (Cenomanian) Ostracode from Wasia Formation of Saudi Arabia. Univ. Kausas. Paleand. Contr. Paper, (108), pp.1-6.

Al-Furiah, A.A.F., 1984a. Maastrichtian Ostracode from the Aruma Formation of Saudi Arabia. Rev.Micropal., 4(3), pp.159-170.

Al-Furiah, A.A.F., 1986. Kaesleria, a new Ostracode genus from the Aruma Formation (Upper Cretaceous) of Saudi Arabia. .Jour. of paleo., 60(3), pp.701-710.

Al-Furiah, A.A.F. and Siddiqui, Q.A., 1981. The Ostracode genus Anommatocythere from middle Eocene of Pakistan and Saudi Arabia. Jour. Coll. Sci. Uni.Riyadh, 12(2), pp.429-441.

Al-Sayyab, A.S., 1956. Cretaceous Ostracode from the Persian Gulf Area. Graduate Thesis, College of the state university of Lowa,138p.

Al-Ubide, W., 1989. Upper Cretaceous Ostracode From well Khasab no.1 Hemeren area, northeastern Iraq. Unpublished Ph.D. Thesis, Mosul Univ., 156p.

Athersuch, J., 1987. Ostracode founas from the Halul,Laffan and Nahr Umr Formations of offshore Abu Dhabi, U.A.E. J.Micropal., 6(1), pp.1-11.

Athersuch, J.,1988. The Biostratigraphy of Cretaceous Ostracode from Oman In:T.Hanai, N.Ikeyo and K.Ishizaki (eds.), proc. the 9th International symposium on Ostracode, held in Shizuoks, Japan, 29 July-2 August 1985, Evolutienary Biology of Ostracode-its fundemental and applications, 187-1206 Kodonsha. Tokyo, Elsevier.

Athersuch, J., 1994. The Biostratigroptic Significance of Cretaceous Ostracode from the Arabian Gulf. In Simmons, M. (ed.). Micropaleontology and Hydrocarbon Exploration in the middle East, Chapman and Hall ,London, pp.253-265.

Apostolescu,V.,1961.Contribution a letude paleontologique (Ostracode)et stratigraphique des bassins Cretaces et tertiaires de 1' Afrique occidentale. Rev. Inst. France, Petrole, 16(7-8), pp.779-867.

Aziz, N.M., 1990. Middle Eocene Ostracode from Dohuk area, north Iraq. M.Sc. Unpubl. Thesis, Mosul Unvi, Iraq, 244p.

Aziz, N.M., 2001. Two new species of the Ostracode genus Buntania Apostolescu of the Paleocene-Eocene from west and north Iraq. Iraqi Jour. Earth Sci., 11(2), pp. 19-37. 
Babinot, J.F. and Bourdillon-de-Grissac,C., 1987. Associations d, Ostracode de 1'AlbienMaastrichtien du Dhofar (Oman) .Affinities Paleobiogeographiques et implications geodynamique. Bull. Soc. Geol. France, (8)5, pp.287-94.

Bassiouni, M.N., 1969a. Einiga Costa- und carinocythereis (Reticulata) Arten aus dem Palazon van Jordanien (Ostracode). N.J.B. Geol. Paleont. Abh., 134(1), pp.1-16.

Bisimuth, H. Boltenhagen, C., Donze, P. Lefever, J. and Saint-Marc, P., 1981. Le Cretace moyen et superienr du Djebel Semmama (Tunisie du centre-Nord); Rech. Explor. Prod. Elf Aquitaine, 5 (2), pp.192-267.

Deroo, G., 1966. Cytheracea (Ostracodes) du Maastrichtien de Maastricht (Pays-Bas) et des regions voisines, resultats stratigraphiques et paleontologiques de leur etude. Geol. Stichting, Med., Ser., C.2(2), pp.1-197.

Grekoff, N., 1951. Quelques Ostracode noaveaux du Senonien superieur du Cameroun Rev. Inst. Franc .Pet. et Ann. Comb. Liq., 6, pp.53-59.

Grekoff, N., 1968. Sur la valeur stratigrphy et les relations paleographiques de quelques Ostracodes du Cretace du paleocene el de Eocene in ferierr al Algeria orientale proe. 3rd Afr. Nic. Coll., 1968, pp.227-248, Cairo: Nat. Int. and Docv. Cent. Grekoff, Resultas geologiques et micropaleontolgiques du sondage d'El krachem (Hauts plateaux algerois). Bull. Soc. Geol. France, Ser.6, 3(4-6), pp.489-491.

Grekoff, N., 1954. Ostracodes. In: G. Cheylan, J. Magne, J.Sigal et N. g Grosdidier, E., 1973. Associations d' Ostracodes du Cretace d'Iran. Rev. Inst. France Petrol., 28 (2), pp.313-169.

Grosdidier, E., 1979. Principaux Ostracodes marins d I' intervalle Aptien-Turonien du Gabon (A frique accidental). Bull. Cont. Rech. Explor-procd. Elf. Aquitaine, 3, pp.1-35.

Hazel, J.E., 1967. Classification and distribution of the recent Hemicytheridea and Trachylelerididae (Ostracodes) off northeastern north America. U.S. Geol. Survey, Prof paper, no.564, pp.1-49.

Khalaf, S.K. and Aziz, N.M., 2001. On some species of the Superfamily Cythracen (Ostracode; Crustacea) from Khurmala Formation, Shaqlawa area of N. Iraq. Iraqi Jour. Earth Sci., 1(1), pp.9-19.

Krommelbein, K., 1964. Ostracoden aus der marien (kusten-kreide) Brosiliens. 1: Brachycythere-Brachycythere)sapucariensis n.sp aus dem Turonium-senckenb lethaea, 45, 6, pp.489-495.

Reyment, R.A., 1960. Studies on Nigerian upper Cretaceous and Lower Tertiary Ostracode.Part 1: Senonian and maastrichtian Ostracode. Stockholm Contr. Geol., 7, 238pp.

Siddiqui, Q.A., 1971. Early Tertiary Ostracode of the family Trachyleberididae from west pakistan. Bull Br. Mus. Nat. Hist. Geol. Suppl., 9, pp. 1-98.

Sohn, I.G., 1970. Early Tertiary Ostracode from west pakistan. Mem. Geol. Surv. Pakistan Paleoa., 3(1), pp.1-91.

Waer, A., 1992. Tertiary and Upper Cretaceous Ostracode from NW offshore, Libya. Their Taxonomy, Biostrigraphy and correlation with adjacent areas. Al-Fateh Univ., Tripoli, Libya., 57, pp.1- 445.

Yousif, M.S., 1987. Middle Eocene-Lower Miocene Ostracode from Sinjar anticline northwest Iraq. M.Sc. Thesis, Mosul Unv., 190p. 
الانتشار الطبقي والاتجاهات الطورية لاوسترلكودا. .

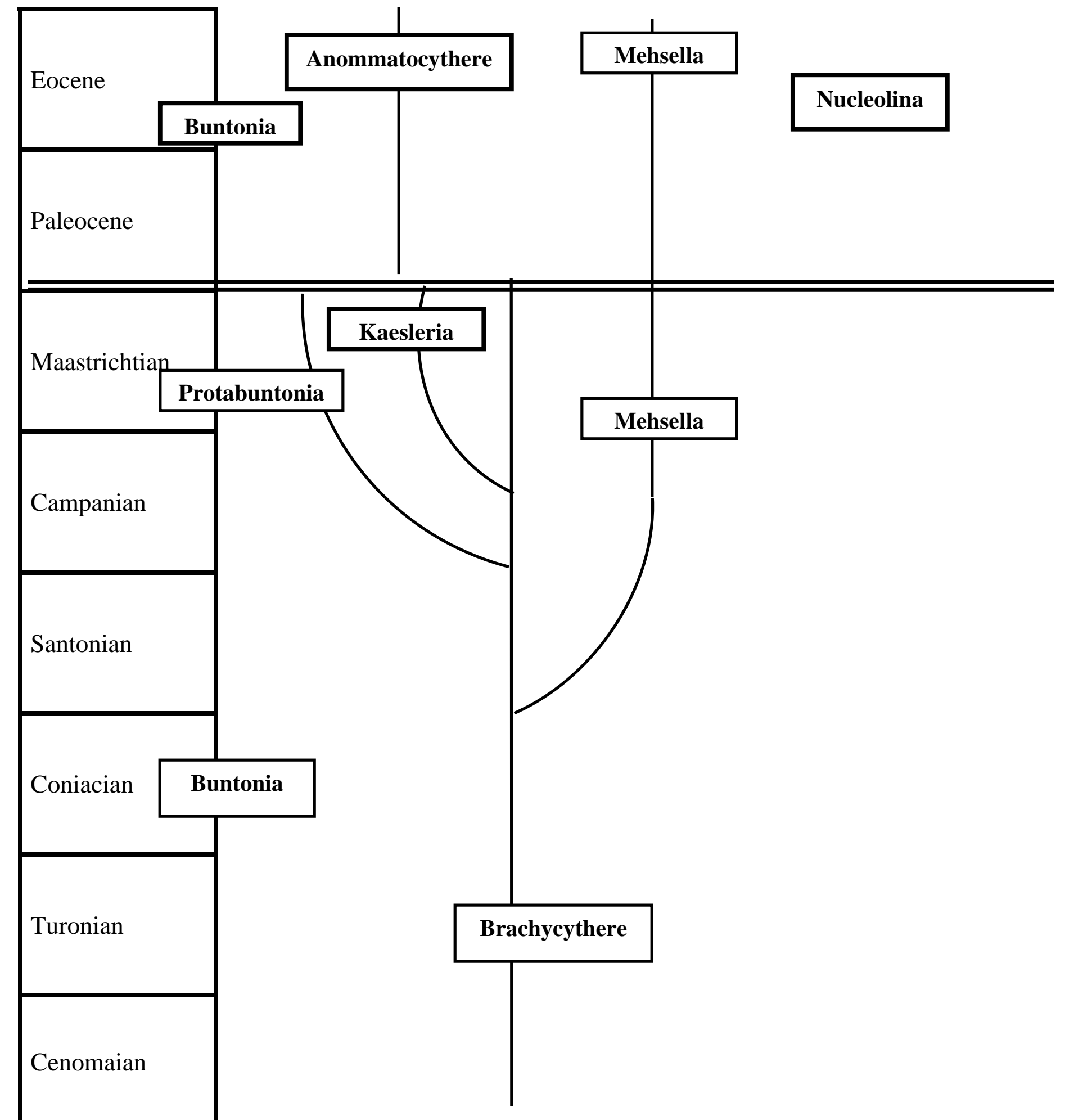

\begin{tabular}{|l|l|l|l|l|l|l|l|l|l|}
\hline \multirow{2}{*}{ Species } & \multirow{2}{*}{ References } & \multirow{2}{*}{ Locality } & \multicolumn{3}{c|}{ Cretaceous } & \multicolumn{3}{c|}{ Tertiary } \\
\cline { 5 - 10 } & & & Con & Sant. & Camp & Maa. & Pal. & Eoc. & Olig. \\
\hline Onia IRE & Grosdidier, 1973 & Irn. & & & & & & & \\
\hline C33 & Grosdidier, 1973 & Irn., O. & & & & & & & \\
\hline
\end{tabular}




\section{نسرين مل اله عزيز}

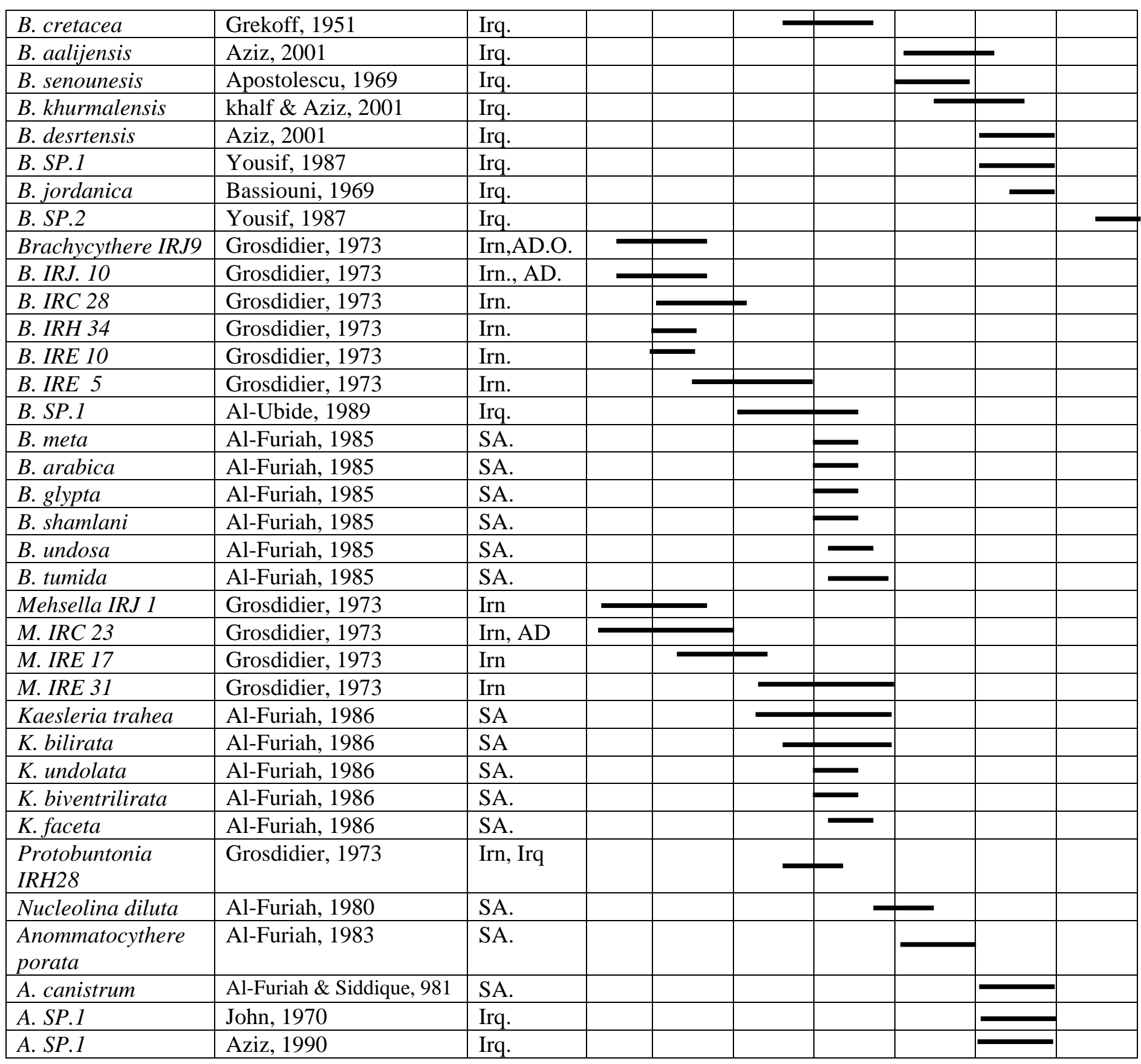

